(DILI) following anti-TB therapy. No such analysis has been performed in the UK.

Aims To assess

1. The prevalence of markers of $\mathrm{HBV}$ and $\mathrm{HCV}$ infection in patients undergoing anti-TB therapy.

2. Whether serological evidence of $\mathrm{HBV} / \mathrm{HCV}$ increases risk of DILI.

Method A prospective study on 429 TB patients receiving anti-TB therapy in a diverse urban TB clinic. Serological markers of HBV/ $\mathrm{HCV} / \mathrm{HIV}$ infection were documented. ALT was measured prior to treatment and 2-4 weeks after treatment initiation. DILI was defined as an ALT rise of greater than 2-times upper limit of normal following a normal pre-treatment level $(<40 \mathrm{IU} / \mathrm{ml})$.

Results $58.7 \%$ of patients were from either South-East Asia or Africa. $61(14.2 \%)$ patients were isolated anti-HBc antibody positive (Anti-HBc), $11(2.6 \%)$ were Hepatitis B surface antigen positive (HBsAg) and $7(1.6 \%)$ were HCV antibody positive. The prevalence of active $\mathrm{HBV} / \mathrm{HCV}$ infection was significantly higher than the estimated UK prevalence in this urban TB clinic. Three $(5.3 \%)$ patients with serological evidence of $\mathrm{HBV} / \mathrm{HCV}$ had DILI compared to $25(9.5 \%)$ of those without.

Conclusion HBV/HCV prevalence is higher among TB patients and routine screening for viral hepatitis should be considered in this group. DILI was not increased in patients with serological markers of $\mathrm{HBV} / \mathrm{HCV}$.

\section{P64 THE EFFECT OF WOOD SMOKE ON THE ABILITY OF HUMAN MACROPHAGES TO PHAGOCYTOSE AND KILL MYCOBACTERIUM TUBERCULOSIS}

doi:10.1136/thoraxjnl-2011-201054c.64

${ }^{1} \mathrm{~J}$ E Scriven, ${ }^{2} \mathrm{D}$ G Fullerton, ${ }^{1} \mathrm{~A}$ Warman, ${ }^{1} \mathrm{~S}$ B Gordon. ${ }^{1}$ Liverpool School of Tropical Medicine, Liverpool, UK; ${ }^{2}$ University Hospital Aintree, Liverpool, UK

Introduction Half of the world's population are exposed to household air pollution from biomass fuels, which are increasingly recognised as a global risk factor for impaired respiratory health. Data from case-control studies has associated Tuberculosis (TB) with biomass fuel exposure. We hypothesised that particulate matter (PM) within alveolar macrophages of individuals exposed to biomass smoke detrimentally affects macrophage function by: (A) Reduced phagocytosis of TB, leading to increased infectivity and (B) Impaired macrophage killing of $\mathrm{TB}$, leading to increased susceptibility to disease. This study assessed the ability of wood smoke exposed human monocyte-derived macrophages (MDMs) to phagocytose and kill TB in vitro.

Methods MDMs, from buffy coats, were cultured in vitro for 12 days and then exposed to suspensions of wood smoke PM (Malawian (MW) and Norwegian (NW) wood, $10 \mu \mathrm{g} / \mathrm{ml}$ or $50 \mu \mathrm{g} /$ $\mathrm{ml}$ ) for $8 \mathrm{~h}$. The PM loaded cells were then challenged with TB. Phagocytosis was assessed by manually counting infected macrophages, using fluorescent microscopy and by quantitative culture of day 1 supernatant and macrophage lysate. Remaining cells were maintained in culture for 7 days before lysis and quantitative culture to assess intracellular growth.

Results Microscopy data showed a reduced proportion of infected macrophages in the high dose NW smoke group (Abstract P64 figure $1 \mathrm{~A})$, and fewer TB bacilli per macrophage in the two MW smoke groups (Abstract P64 figure 1B). Quantitative culture of day 1 supernatant and cell lysate showed no difference between the groups (Abstract P64 figure 1C). Quantitative culture of day 7 lysate showed reduced Mycobacteria loads in the high dose NW smoke group (Abstract P64 figure 1D).
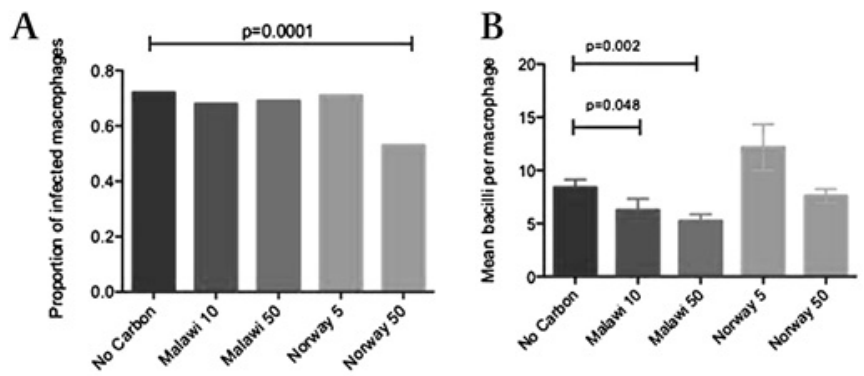

C
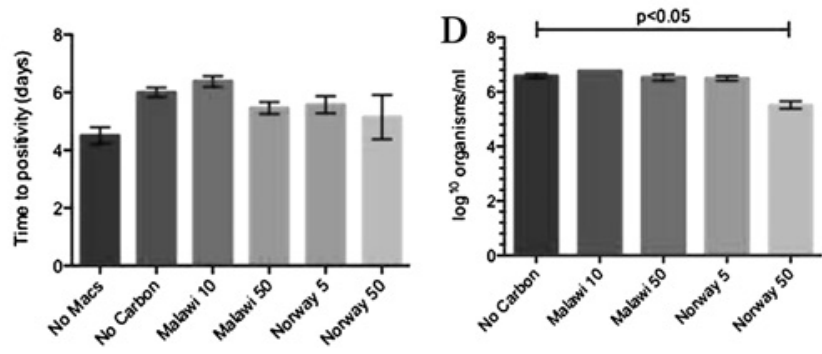

Abstract P64 Figure 1 The effect of wood smoke on monocyte-derived macrophages to phagocytose and kill Mycobacterium tuberculosis: (A) Effects of wood smoke on macrophage phagocytosis of TB (assessed by microscopy). (B) Mean numbers of bacilli per infected macrophage. (C) Mean time to positivity for culture of day 1 supernatants. (D) Calculated Mycobacterial load of day 7 lysates (log scale).

Discussion We have developed an in vitro model to assess the interaction between TB and wood smoke exposed macrophages. Phagocytic data does not fully support increased TB infectivity in biomass smoke exposed individuals. Quantitative culture data does not demonstrate a difference in the ability of wood smoke exposed MDMs to kill TB; high dose NW smoke led to a reduced intramacrophage growth of TB probably as a result of the toxic effect of PM on Mycobacteria growth. Modifications of this model are required to provide mechanistic evidence of an association of $\mathrm{TB}$ with wood smoke exposure.

\section{P65 TREATING TUBERCULOSIS IN RURAL SOUTH AFRICA} doi:10.1136/thoraxjnl-2011-201054c.65

J N Periselneris. Church of Scotland Hospital, Tugela Ferry, KwaZulu-Natal, South Africa

Methods All the patients admitted to the male and female Tuberculosis wards at a rural hospital in KwaZulu-Natal, South Africa, during a 1-month period were entered into a database. Basic demographic data were collected as well as information about HIV status, medications, complications and 1-month mortality. The area's tuberculosis (TB) incidence is 1046 per 100000 and there is an HIV infection rate of $29 \%$.

Results 70 patients were admitted during the time allocated. $89 \%$ were HIV positive. The mean CD4 count of the HIV positive patients was 114 , however only $45 \%$ of patients were on ARVs at time of admission. $21 \%$ of patients had extrapulmonary TB. Only $17 \%$ were AFB positive, a further $9 \%$ had culture results back, two patients cultured proven multidrug resistant TB. Two patients had a history of completed XDR TB treatment. 10\% patients developed drug induced hepatitis, $3 \%$ had pneumothoraces, $10 \%$ had coexistant renal impairment, $11 \%$ had neurological complications. There was a $23 \% 1$-month mortality. No patients who were HIV negative died. $27 \%$ died of respiratory failure, $12 \%$ had hepatic failure, likely secondary to antituberculous medication. 